\title{
Evaluación del programa de introducción a la investigación científica en la residencia del Hospital General de Niños Pedro de Elizalde, 1997-2012
}

\author{
Evaluation of the program of introduction to research in pediatric residency at \\ Hospital General de Niños Pedro de Elizalde, 1997-2012
}

\author{
Dr. Raúl Cimbaro Canella ${ }^{a}$ Prof. Verónica Gatto Bellora ${ }^{a}$, Dra. María Liliana Yazde Puleio ${ }^{a}$ \\ Dr. Fernando Adrián Torres ${ }^{a}$ Dra. María Fabiana Ossorio y Dr. Fernando Ferrero ${ }^{a}$
}

\section{RESUMEN}

Introducción. Existe poca información sobre investigación en la residencia. Nuestro objetivo es describir la producción científica de residentes, estimar la tasa de difusión (presentación en evento y /o publicación) de sus trabajos eidentificar potenciales barreras para ella.

Métodos. Estudio transversal, que incluyó todos los trabajos del Programa de Capacitación en Investigación para Residentes (Hospital General de Niños Pedro de Elizalde, HGNPE), 19972012.

Resultados. Contactamos a los responsables de 451/531 proyectos. La producción anual se incrementó sostenidamente $\left(1997=12\right.$ vs. $\left.2012=40 ; R^{2}=0,88 ; p<0,001\right)$.

Considerando exclusivamente proyectos de investigación (sin incluir revisiones) ( $n=195), 30,8 \%$ alcanzaron difusión $(26,7 \%$, presentación; y 11,3\%, publicación).

Alcanzaron difusión más frecuentemente las investigaciones retrospectivas que las prospectivas ( $37,5 \%$ vs. $21,7 \%$; OR: 2,17 ; $\mathrm{p}<0,02)$ y las analíticas más que las descriptivas $(41,2 \%$ vs. 16\%; OR: 3,$67 ; \mathrm{p}<0,001)$.

La falta de tiempo fue el motivo más frecuente $(45,9 \%)$ para no alcanzar la difusión.

Conclusión. El30,8\% de los proyectos de investigación alcanzó difusión. Tuvieron más posibilidades aquellos con recolección retrospectiva de datos y diseño analítico.

Palabras clave: internado y residencia, publicaciones, proyectos de investigación, informe de investigación.

http:/ /dx.doi.org/10.5546/aap.2015.337

a. Docencia e Investigación, Hospital General de Niños Pedro de Elizalde.

Correspondencia:

Dr. Raúl Cimbaro Canella, raulcc21@gmail.com

Financiamiento: Ninguno.

Conflicto de intereses: Durante los últimos 10 años, Laboratorios Roemmers becó a los autores del mejor trabajo de investigación originado en el programa para presentarlo en la Sociedad Latinoamericana de Investigación Pediátrica (SLAIP), sin participar en la selección de los ganadores.

Recibido: 23-12-2014

Aceptado: 2-3-2015

\section{INTRODUCCIÓN}

A pesar de que la investigación es parte fundamental de la profesión médica y, por lo tanto, su enseñanza en el posgrado debería tener el mismo rigor que la de otros conocimientos, esto dista de la realidad. En 1996, solo 27\% de los programas de residencia de Estados Unidos incluían actividades de investigación. ${ }^{1}$

Además, aunque ninguna actividad de investigación puede darse por concluida hasta que sus resultados sean difundidos y sometidos al escrutinio de pares, solo una limitada proporción alcanza la publicación..$^{2,3}$ Es posible que esta realidad sea más evidente en aquellas desarrolladas durante la residencia.

Existen diversas estrategias para la enseñanza de la investigación en el posgrado inmediato. ${ }^{4}$ El Hospital General de Niños Pedro de Elizalde (HGNPE), desde hace 18 años, ha adoptado un programa integral con tutores. ${ }^{5} \mathrm{~A}$ pesar de que la producción científica del Hospital se ha incrementado desde su puesta en práctica, ${ }^{6}$ no se ha valorado aquella ligada específicamente al programa.

El objetivo del presente trabajo es describir la producción científica de residentes de un hospital pediátrico, estimar la tasa de difusión (presentación/publicación) de sus trabajos e identificar potenciales barreras para ella.

\section{MÉTODOS}

Diseño: Transversal.

Población: Todos los trabajos científicos del Programa de Capacitación en Investigación para Residentes (HGNPE), desde 1997 a 2012.

Procedimientos del estudio: De los registros del Comité de Docencia e Investigación (CODEI), se identificaron los trabajos del programa y sus autores. Se contactó un responsable por proyecto (correo electrónico, teléfono o personalmente) y se le solicitó que participara de una encuesta autoadministrada y cerrada, que indagaba sobre 
el estado de la investigación (finalizada, en curso, suspendida), la difusión de los resultados (presentados y/o publicados) y, cuando correspondía, los motivos para no alcanzar la difusión.

Se consideró que la investigación fue presentada cuando sus resultados se hubieran dado a conocer en una reunión científica local, nacional o internacional (fuera de la institución), y publicada, cuando apareciera en una publicación periódica científica con ISSN.

De los registros del CODEI, se consignaron los siguientes:

- Residencia: Especialidad del autor.

- Tipo de proyecto: Revisiones o investigaciones, y estas últimas con recolección de datos retrospectiva o prospectiva.

- Diseño: Sin incluir las revisiones, las investigaciones (con recolección de datos retrospectiva o prospectiva) se clasificaron en descriptivas y analíticas.

Análisis estadístico: Las variables categóricas se expresan como porcentajes con intervalos de confianza del 95\%. Se exploró la asociación entre difusión (presentación/publicación) y tipo de investigación, diseño y año de desarrollo mediante chi cuadrado. Las variables mencionadas se incluyeron en un modelo de regresión logística. Nivel de significación: $\mathrm{p}<0,05$. Se utilizó SPSS 11.5 (SPSS Inc).

Consideraciones éticas: Se solicitó y obtuvo aprobación de los Comités de Ética en Investigación y Docencia e Investigación del Hospital. La investigación fue registrada en el Registro de Proyectos de Investigación del GCBA (99/13).

\section{RESULTADOS}

Se identificaron 531 proyectos generados en el programa entre 1997 y 2012, y se logró contactar a los responsables de 451 ; todos estaban finalizados. El 81,1\% correspondían a residentes de la especialidad de pediatría y $56,8 \%$ eran revisiones (Tabla 1).

La producción anual se incrementó significativamente desde el inicio del programa $\left(1997=12\right.$ vs. $\left.2012=40 ; R^{2}=0,88 ; p<0,001\right)$ (Figura 1).

Al considerar exclusivamente proyectos de investigación (sin incluir revisiones) $(n=195)$, $30,8 \%$ alcanzaron difusión (26,7\% presentados y $11,3 \%$ publicados). La proporción de proyectos de investigación que alcanzó difusión se incrementó significativamente en la segunda mitad del período estudiado $(2005-2012=36,6 \%$ vs. 1997 $2004=18,03 \%$; OR= 2,6 IC 95\%: 1,2-5,5; $\mathrm{p}=0,01$ ).

Los proyectos de investigación con captura de datos retrospectiva tuvieron más posibilidades de alcanzar difusión que los prospectivos $(37,5 \%$ vs. $21,7 \%$; OR=2,17 IC 95\%:1,13-4,14; $\mathrm{p}<0,02)$. Los proyectos con diseño analítico tuvieron más posibilidades de alcanzar difusión que los descriptivos (41,2\% vs. $16 \%$; OR=3,67 IC $95 \%$ : 1,8 $7,4 ; \mathrm{p}<0,001)$.

Luego de controlar por momento del programa en que se realizó (primera o segunda mitad del período), tipo de recolección de datos retrospectivo y diseño analítico, se mantuvieron como predictores independientes de difusión (Tabla 2).

Los resultados de 135 proyectos nunca fueron difundidos. La falta de tiempo (45,9\%) fue referida más frecuentemente como motivo del fallo en alcanzar la difusión, seguida de falta de interés $(13,3 \%)$, falta de estímulo $(13,2 \%)$ y otras causas $(27,6 \%)$.

\section{DISCUSIÓN}

Diversas estrategias han sido diseñadas para incrementar la actividad científica en la residencia,

Tabla 1. Distribución de los proyectos según tipo y diseño

\begin{tabular}{llcc}
\hline Tipo & & Proyectos $(\mathbf{n = 4 5 1 )}$ & \% \\
\hline Revisión bibliográfica & & 256 & 56,8 \\
Observacionales & Descriptivo & 80 & 17,7 \\
& Transversal & 84 & 18,6 \\
& Casos y controles & 4 & 0,9 \\
& Cohorte & 9 & 2,0 \\
Experimentales & Experimental no farmacológico & 11 & 2,4 \\
& Experimentación básica & 1 & 0,2 \\
& Experimental fase 3 & 1 & 0,2 \\
& Experimental fase 4 & 5 & 1,1 \\
\hline
\end{tabular}


que incluyen el desarrollo de un proyecto de investigación entre todos los residentes, ${ }^{7}$ rotación específica en el área de investigación ${ }^{8}$ y premios a la mejor producción científica. ${ }^{9}$ Con compromiso institucional, habitualmente, las diferentes acciones han tenido éxito, pero su mantenimiento en el tiempo es fundamental para lograr un "cambio cultural". 9

La incorporación de la investigación como objetivo curricular de la residencia de pediatría y el decidido respaldo institucional seguramente son responsables del incremento de la producción científica en nuestra institución, donde el programa fue sostenido ininterrumpidamente durante 15 años, lo que da la impresión de que se ha logrado o se encuentra en camino de lograr ese "cambio cultural". Por un lado, la totalidad de proyectos de investigación del programa relevados fueron finalizados. Por otro, la producción científica de la residencia, casi inexistente antes de la implementación del programa, se incrementó de manera significativa con el correr de este, a pesar de que se mantuvo prácticamente constante la dotación de residentes. Más aún, es posible que haya tenido influencia en el incremento de la producción científica de la institución verificada en los últimos años. ${ }^{10}$

No sorprende este resultado, ya que existe evidencia de que programas con algunas características similares al nuestro pueden incrementar significativamente la producción científica de los residentes. ${ }^{11}$

Una evaluación aparte merece la tasa de difusión de los trabajos desarrollados en el programa. Es una preocupación constante que los resultados de las investigaciones alcancen difusión entre los pares, únicos responsables de validarlos. Encontramos que 30,8\% de los proyectos de investigación alcanzaban difusión (presentación/publicación); específicamente la tasa de publicación alcanzó 11,3\%. Este valor se encuentra muy por debajo del $36 \%$ reportado por Holmes con un programa similar para residentes de emergentología. ${ }^{12}$ Sin embargo, la diferencia es similar a la que existe entre la tasa de publicación de trabajos presentados en congresos

Figura 1. Proyectos de investigación según tipo y año

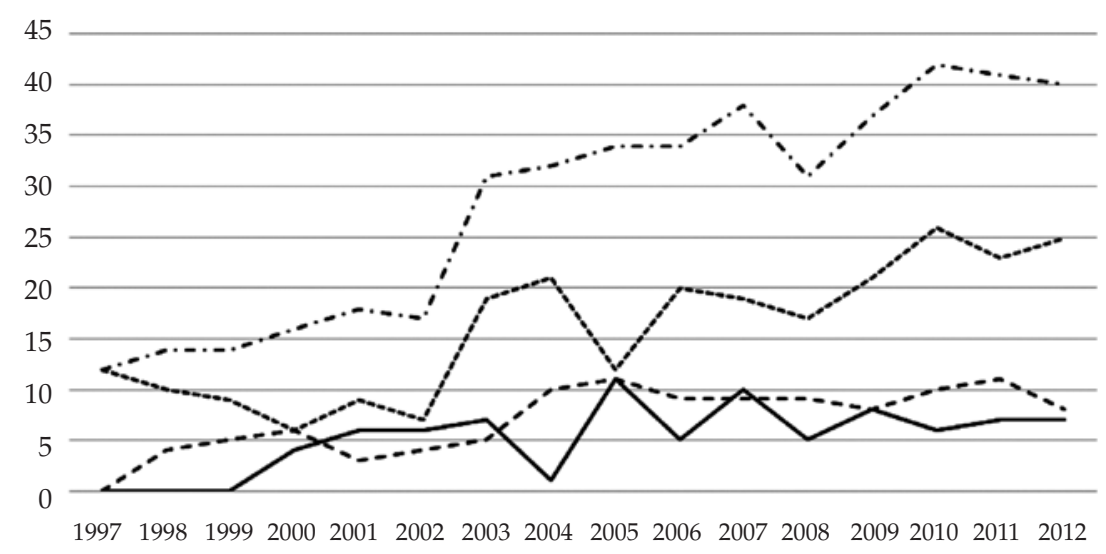

------Revisión - - - Retrospectivo _-Prospectivo -.-.-Total

TABLA 2. Análisis multivariado que incluye potenciales predictores de difusión (presentación/publicación) de los proyectos

\begin{tabular}{lcccc} 
& OR & \multicolumn{2}{c}{ IC 95\% } & p \\
\hline Momento de realización (> 2004) & 2,179 & 0,987 & 4,809 & 0,054 \\
Diseño (analítico) & 3,705 & 1,767 & 7,766 & 0,001 \\
Tipo (retrospectivo) & 2,781 & 1,387 & 5,574 & 0,004 \\
\hline
\end{tabular}

Test de Hosmer-Lemeshow, $\mathrm{p}=0,378$.

OR: odds ratio. IC 95\%: intervalo de confianza 95\%. 
en el hemisferio norte $(44,5 \%)^{3}$ y la referida para trabajos presentados en congresos argentinos de pediatría $(11,4 \%) \cdot{ }^{13}$ Es muy posible que, en nuestro medio, la publicación científica, punto final deseado para toda investigación, no tenga el mismo prestigio ni reciba la misma prioridad en el desarrollo de una carrera profesional.

Sin embargo, es importante destacar que, cuando al esfuerzo del programa se une una recompensa, pueden lograrse mejores resultados. ${ }^{9}$ Aunque escapa al objetivo de este trabajo, cabe destacar que nuestro programa selecciona cada año el mejor trabajo y beca a sus autores para presentarlo en la Reunión Anual de la Sociedad Latinoamericana de Investigación Pediátrica. En los 16 años estudiados, han sido becados 13 trabajos, 9 de los cuales alcanzaron la publicación en forma completa $(69 \%)$.

$\mathrm{Al}$ intentar analizar la posible causa de fallar en difundir los resultados, no llama la atención que los estudios analíticos alcanzaran con más frecuencia la difusión que los meramente descriptivos; sin embargo, puede sorprender que esto haya ocurrido con los retrospectivos en relación con los prospectivos. Es muy probable que esto último se deba a la mayor facilidad para acceder a los datos en los retrospectivos, lo que permite manuscritos más sólidos y, consecuentemente, con mayor probabilidad de ser difundidos.

La falta de tiempo fue referida como causa más frecuente $(45,9 \%)$ para no difundir resultados, una frecuencia superior a la referida habitualmente $(33 \%) .{ }^{14}$ Es posible que la carga horaria y la dedicación que exige el programa de residencia limite aún más el tiempo disponible para esta actividad.

El estudio presenta potenciales limitaciones. Por un lado, la notificación de presentación / publicación era referida por los autores; sin embargo, los datos fueron verificados en publicaciones y libros de resúmenes de congresos. Aunque no se logró contactar a los autores de todos los trabajos, la muy buena tasa de respuesta $(85 \%)$ permite inferir que no existe un sesgo importante. Por otro lado, el estudio se llevó a cabo en 2014 e incluyó proyectos finalizados hasta 2012. Aunque existe evidencia de que la mayor parte de los trabajos se publican dentro de los 2 años de finalizados, ${ }^{15}$ podríamos haber subestimado la tasa de publicación, especialmente si se tiene en cuenta que esta fue mayor en los últimos años del período en estudio.

\section{CONCLUSIÓN}

El 30,8\% de los proyectos de investigación de los residentes alcanzó difusión; los proyectos con recolección retrospectiva de datos y diseño analítico tuvieron más posibilidades de hacerlo. La falta de tiempo fue el motivo para no alcanzar difusión más referido.

El incremento de la producción científica de los residentes y de la difusión de sus trabajos respalda el desarrollo del programa.

\section{REFERENCIAS}

1. Brouhard BH, Doyle W, Aceves J, McHugh MJ. Research in pediatric residency programs. Pediatrics 1996;97(1):71-3.

2. Von Elm E, Constanza MC, Walder B, Tramer MR. More insight into the fate of biomedical meeting abstracts: a systematic review. BMC Med Res Methodol 2003;3:12.

3. Scherer RW, Langenberg P, Von Elm E. Full publication of results initially presented in abstracts. Cochrane Database Sys Rev 2007;(2):MR0000005.

4. Penrose LL, Yeomans ER, Praderio C, Prien SD. An incremental approach to improving scholarly activity. J Grad Med Educ 2012;4(4):496-9.

5. Manjarin M, Cutri A, Noguerol E, Torres F, et al. Enseñanza de la investigación con un sistema de tutores durante la residencia de pediatría. Arch Argent Pediatr 2007;105(4):336-8.

6. Domínguez P, Chiolo MJ, Davenport MC, Di Lalla S, et al. Evaluación de la producción científica del Hospital General de Niños Pedro de Elizalde, 2000-2011. Arch Argent Pediatr 2014;112(2):147-52.

7. Shin AY, Almond CS, Mannix RC, Duncan CN, et al. The Boston Marathon Study: a novel approach to research during residency. Pediatrics 2006;117(5):1818-22.

8. Kanna B, Deng C,EricksonSN, ValerioJA, etal. The research rotation: competency-based structured and novel approach to research training of internal medicine residents. $B M C$ Med Educ 2006;6:52.

9. Rothberg MB, Kleppel R, Friderici JL, Hinchey K. Implementing a resident research program to overcome barriers to resident research. Acad Med 2014;89(8):1133-9.

10. FerreroF, Ossorio MF, Eiguchi K. La investigación científica en las instituciones de salud del Gobierno de la Ciudad de Buenos Aires. Medicina (B Aires) 2013;73(5):443-7.

11. Kurahara DK, Kogachi K, Yamane M, Ly CL, et al. A pediatric residency research requirement to improve collaborative resident and faculty publication productivity. Hawaii J Med Public Health 2012;71(8):224-8.

12. Holmes JF, Sokolove PE, Panacek EA. Ten-year experience with an emergency medicine resident research project requirement. Acad Emerg Med 2006;13(5):575-9.

13. Canosa D, Ferrero F, Melamud A, Otero PD, et al. Publicación completa de trabajos presentados en el $33^{\circ}$ Congreso Argentino de Pediatría y análisis de factores que impidieron su publicación. Arch Argent Pediatr 2011;109(1):56-9.

14. Song F, Loke Y, Hooper L. Why are medical and healthrelated studies notbeing published? A systematic review of reasons given by investigators. PLoS One 2014;9(10):e110418.

15. Gregory TN, Liu T, Machuk A, Arneja JS. What is the ultimate fate of presented abstracts? The conversion rates of presentations to publications over a five-year period from three North American plastic surgery meetings. Can J Plast Surg 2012;20(1):33-6. 